\title{
El valor cosmocéntrico, estético y del conocimiento en la lengua Quichua del Ecuador
}

\author{
The cosmocentered, aesthetic and knowledge value in the Quichua language of Ecuador
}

Josefina Aguilar Guamán ${ }^{1}$

\section{Resumen}

El quichua es un elemento sustancial de la cultura, producto socialmente configurado, en el que la aprehensión del mundo se manifiesta de forma distinta de las otras culturas. Aunque en este artículo no recurra a comprobar el condicionamiento social del lenguaje como eje de investigación, procuro abordar los significados guardados en las palabras, como medio para acercarme a los modos y formas de pensar y vivir de la cultura quichua en el ánimo y condiciones psíquicas del ser humano, así como la percepción de las matemáticas. Son temas abordados en este artículo, a través de los cuales podemos adentrarnos a entender las diferencias del pensamiento en los Andes y el valor cotidiano que este alcanza hasta la actualidad.

Palabras-clave: Epistemología andina, estética del quichua, espiritualidad andina, etnomatemática.

\begin{abstract}
The Quichua language is an essential element of culture, a socially configured product, in which the apprehension of the world is expressed in a different way in the cultures. Although in this article I do not try todemonstrate the social condition of language as the axis of investigation I try to explain the meanings kept in words, as a means to approach ways of thinking and living of the Quichua culture, both in the courage and psychic conditions of the human being. The perception of mathematics is a topic to be discussed in this article by which we can understand the differences of thought in the Andes and the daily value it reaches up to this time.
\end{abstract}

Keywords: Andean epistemology, aesthetics of quichua, Andean spirituality, ethnomathematics.

\section{Introducción}

El hecho de que las culturas pueden pensarse a sí mismas e intervenir en su destino, las sitúa en el mundo de forma emancipada con capacidad de decidir y desarrollar sus prácticas de vida según sus rasgos distintivos de carácter ontológico, axiológico, epistemológico, de ejercicio de autoridad y organización de la sociedad. Pensarse culturalmente pone en valor a formas de vivir sojuzgadas y las lleva a tomar conciencia de sus raíces de identidad, de su capacidad creativa en torno a la realidad, como seres humanos cognitivos y afectivos.

\footnotetext{
${ }^{1}$ Docente-investigadora de la Universidad Nacional de Educación, UNAE. Mujer perteneciente al pueblo quichua otavalo, de idioma materno quichua. Maestría en Ciencias Políticas y Administración Pública, en la Pontificia Universidad Católica de Quito. Email: huacsara@ hotmail.com.
} 
La reflexión sobre la realidad y las relaciones que establecieron las culturas con lo circundante responden a dinámicas témporo-espaciales locales, las cuales son determinadas por la geografía, los hechos históricos y sociales en las que se han generado; además, la capacidad de pensar sobre sí mismas les permite construir dinámicas de participación e intervención en la realidad a través del tiempo-espacio, y también desde las alteridades.

En las lenguas se puede apreciar la capacidad intelectiva de las diversas culturas sobre la realidad, de modo que creer que existe una manera homogénea de mirarla atenta sobre el contenido creativo del pensamiento humano. La producción del pensamiento en las sociedades está determinada por una necesidad o un deseo de comprender el mundo que les circunda. En la lengua quichua destacan eventuales diferencias sobre la visión del mundo, se acuñan en ella, de manera algo diferente, los significados de la realidad. "Puesto que los hombres utilizan distintos sistemas lingüísticos, que a menudo revelan una morfología y sintaxis fundamentalmente diferentes, corresponden a un círculo cultural distinto y tienen otra teoría" (Schaff, 1967, 90).

Los significados de la lengua quichua encuentran sentido en el desarrollo de relaciones armoniosas con el cosmos, la sociedad y lo espiritual; no tienen sentido en modos intelectuales de pensar y de vivir antropocéntricos, individualistas y extractivistas.

(...) el lenguaje de una comunidad humana dada, que habla y piensa en esa lengua, es el organizador de su experiencia y configura su "mundo" y su "realidad social" gracias a esa función. Formulado de modo distinto y más breve, este pensamiento dice que en cada lenguaje se halla una concepción particular del mundo. (Schaff, 1967, 99)

El quichua es un elemento sustancial de la cultura, producto socialmente configurado, en el que la aprehensión del mundo se realiza de forma distinta. Aunque en este artículo no recurra a comprobar el condicionamiento social del lenguaje como eje de investigación, procuro abordar los significados guardados en las palabras, como medio de acercamiento a los modos y formas de pensar y vivir de la cultura quichua. Así, el valor cosmocéntrico de la vida, el valor estético de la lengua, los estados de ánimo y condiciones psíquicas en el ser humano, la percepción de la matemática que se encuentra elaborada en este idioma, son aspectos que nos brindan una entrada para entender las diferencias del pensar en los Andes y el valor cotidiano que este pensamiento alcanza hasta la actualidad.

\section{El valor cosmocéntrico de la vida en la lengua quichua}

El pensamiento quichua desarrolló el valor cosmocéntrico de la vida; ello tiene coherencia en la manera como los quichua hablantes entendieron a la divinidad, la naturaleza y los fenómenos que se producen en ella. Los pakariy uku yuyaykuna (mitos de origen), los unanchakuna (símbolos), los ñawpa shuyukuna (dibujos rupestres), la ayllu kawsay (vida de los pueblos) y su lengua quichua son un arsenal viviente de esta forma de reflexionar la realidad. Para la filosofía occidental las primeras explicaciones sobre las cosas que le preocupaban, que dieron origen a culturas como las mestizas, constituyeron una manera 
infundada de responderse sobre la realidad; esta se vio como sujeto de interpretación mitológica y no de naturaleza racional, por ende, no demostrativa.

Para Occidente la filosofía se inicia desde Tales de Mileto (625 a.C.), quien planteó el problema de la verdad respecto al origen de las cosas a través de su famosa pregunta: ¿cuál es el principio de todas las cosas? A partir de ella y la reflexión desarrollada en torno a su respuesta, el mundo griego y europeo en general fomentaría el fortalecimiento de la razón como eje conductor del desarrollo de Occidente, de carácter técnico y científico. Así, los pensadores como Sócrates, Platón, Aristóteles vivieron sus vidas en procura de responder esa interrogante dando un comienzo a la filosofía y con ello a aquel que se dedicara a contestar dicha pregunta con base en la razón se lo llamaría filósofo. Se construye de este modo la apertura al entendimiento del mundo por parte de los pensadores griegos, propiciando una aurora al mundo de la verdad, catalogada como universal.

El ejercicio intelectivo del ser humano de explicarse la existencia de la que forma parte, nos lleva al estudio del pensamiento filosófico presente en las diferentes culturas del mundo, por cual es pertinente preguntarnos y cuestionarnos ¿por qué una manera de reflexionar o hacer filosofía debe constituir una verdad universal?, ¿por qué la comprensión de la naturaleza como elemento inerte debe ser una comprensión homogénea en el mundo?, ¿por qué la naturaleza tiene que ser el objetivo final del ser humano y de la sociedad?, ¿por qué el Dios de la Biblia, por ejemplo, y la manera de vivir que él impone debe ser una verdad absoluta? El calentamiento del clima en la Tierra, la extinción de los animales, la sequía de los campos y los bosques, la falta de alimentos en el mundo, entre otros, son aspectos que cuestionan una manera de habitar y de relacionarse las personas con el mundo y que permiten actuar de forma crítica sobre patrimonios epistemológicos, axiológicos y ontológicos que, concretamente, son propios de las culturas industrializadas.

\section{La Pachamama}

En la cultura quichua el pensamiento o la reflexión sobre lo circundante se caracteriza por resaltar el valor cosmocéntrico de la vida, en forma relacional y multidimensional con el tiempo-espacio. Pacha es la voz quichua que recoge el significado cosmocéntrico de la vida.

Pachamama es el tiempo-espacio femenino sagrado, nombra a la procreadora y a aquello donde continúa la vida. "Cada vida tiene un espacio y un tiempo dentro de la Pacha-Mama" (Cachiguango, 2007, 20). Es naturaleza y deidad.

Pacha recoge la multidimensionalidad del espacio conformado por la dimensión estelar, telúrica, y del otro tiempo; expresa todo, como son el cielo, el fuego, el agua, la tierra, los animales, las plantas, los astros. Dentro de la dimensión estelar se encuentran los astros del cosmos; en la dimensión telúrica se incluye al paisaje de los Andes, que está muy ligado al sara tarpuy, siembra del maíz, con los raymikuna o celebraciones, es decir que se trata de un concepto agrocéntrico-estelar del tiempo. El "otro tiempo" es entendido como el de la trascendencia, donde la vida después de la vida es posible. La dimensión del tiempo se entiende como el muyиy, movimiento, unido indisolublemente al espacio; condición 
existencial infinita de la cual depende la vida en el cosmos, de la que los miembros del ayllu, familias andinas, son conscientes y por la que viven en relaciones permanentes de reciprocidad con la multidimensionalidad de la pacha.

El principio ordenador del pensamiento quichua lo encontramos en el valor cósmico de la vida. El ser humano de los Andes desarrolló una reflexión profunda de la pacha, tiempo-espacio; se podría decir que la idea fundante de su pensamiento partió del razonamiento de este concepto; la búsqueda de respuestas y la solución de problemas partió de la reflexión de esa unidad inseparable. Maravillosamente, los abuelos y las abuelas en uso de esta lengua trascendieron del proceso reflexivo que los llevó a observar la naturaleza y todo lo que ocurre en ella, hacia abstracciones filosóficas y a abordarlas dentro de categorías prácticas; nos condujeron a entender las leyes de la física del mundo, de un modo afectivo y cognitivo a la vez.

La comprensión de la pacha como unidad inseparable se pone en práctica en el dicho de los yuyak", "Tukuymi ishkay, ishkay": todo en el mundo es de dos en dos; "Tukuymi kawsan": todo está vivo. Son principios ordenadores que ponen en resguardo o encierran todas las formas de existencia del cosmos.

"Tukuymi ishkay, ishkay", "Tukuymi kawsa” se han transmitido con la tradición oral, con las que los padres han enseñado a los más jóvenes, una manera de relacionarse con todo lo que nos rodea bajo un ordenamiento natural que trasciende a lo social, administrativo, normativo de una comunidad. Dentro de esta lógica es que la relacionalidad se produce, constituyéndose en principios filosóficos que organizan la práctica convivencial del ser humano quichua con todas las formas de existencia.

El pensamiento no puede ser chulla, (shuklla) unitario, individual, también debe corresponderse con el principio de paridad. En los Andes la dualidad del pensamiento siempre ha sido una enseñanza viva. Yuyana, palabra quichua que los yuyak han transmitido como la comprensión de "pensar", al parecer expresa esa paridad del pensamiento, al tener como significado pensar junto con el sentir.

El pensamiento reflexivo runa ${ }^{3}$ nunca se separó del valor cósmico de la vida, como ocurrió en Occidente con los iniciadores del pensamiento filosófico griego, que atribuyeron un carácter racional a la naturaleza, para dar explicación al origen del "todo", en procura de profundizar el logos (conocimiento). Su preocupación fundante fue dar respuesta a las primeras causas a través de encontrar el "arjé", y para ello los filósofos, en el intento de racionalizar el origen del mundo, argumentaron que el agua, el fuego, el aire, como los elementos de los cuales se inició la existencia, son de carácter material únicamente, carentes de sami, es decir de energía o de vida. En tiempos de los Incas los amautas ${ }^{4}$ avanzaron en la producción del derecho, astronomía, medicina, ingeniería, orientados por el valor

\footnotetext{
${ }^{2}$ Abuelas, abuelos y sabios en idioma quichua.

${ }^{3}$ Ser humano, en idioma quichua.

${ }^{4}$ Sabios, filósofos, maestros, poetas, que asesoraban en la toma de decisiones del Inca.

Zetetiké, Campinas, SP, v.26, n.1, jan./abr. 2018, p.8-20
} 
cosmocéntrico de la vida; desarrollaron la voluntad y la capacidad intelectual de una manera equilibrada y en armonía con la Pachamama.

\section{Los raymikuna, celebraciones a la multidimensionalidad del cosmos}

Durante el año andino, los raymikuna, celebraciones del calendario agrofestivo, constituyen ritos de reciprocidad a la multidimensionalidad del cosmos. El cosmos se entiende como un ser vivo y organizado de dos en dos; los principios tukuymi kawsa, tukuymi ishkay ishkay son ejes transversales de la simbología andina, que se manifiestan en la ritualidad de los raymikuna. La siembra en la chakra ${ }^{5}$ constituye el acto humano de mayor importancia, donde se pone de manifiesto la comunión con la tierra, como un ser de carácter témporo-espacial, a la que se le debe culto y respeto por su capacidad procreadora de vida.

Tarpuypacha, o tiempo de siembra, es el inicio de esta actividad. Es la celebración al tiempo, al tiempo fecundo, que es posible gracias a la lluvia, al agua como ser femenino. La tierra y el tiempo (de lluvia) constituyen un todo vivo, pacha, al que se debe ofrendar con alimentos de la misma tierra.

Los ayllu ofrecen alimentos dados en la misma chakra como el fréjol, papa, mote, camote y otros productos, al igual que la chicha de maíz. En algunas comunidades, como las del pueblo otavalo, pueblo quitu-cara, las celebraciones a la siembra son identificadas como kuya raymi. Dependiendo de los pueblos y sobre todo de la presencia de las lluvias, los meses de siembra son considerados a partir de septiembre hasta noviembre en algunos pueblos como los cañaris y saraguros, por ejemplo.

Pallaypach, es entendido como el tiempo de recolección de granos, que lo identificamos en dos momentos. El primer momento es cuando los granos sembrados se pueden consumir como alimentos tiernos. Nuevamente el tiempo, la tierra, gracias a la presencia de la lluvia, ha permitido florecer los frutos; los alimentos que se ofrece provienen de la misma chakra, con la diferencia de que serán los productos de temporada, por lo tanto, granos tiernos como las habas, el choclo, las arvejas y la chicha. Dependiendo del mes de siembra, los tiempos de cosecha de granos tiernos pueden darse a partir de febrero, hasta abril en algunos pueblos. La celebración de los carnavales, o pawkar raymi, la preparación de la uchukuta, similar a la fanesca, coinciden con el primer pallaypacha. Actualmente el pawkar raymi es una celebración asociada al "día del sol recto", que se produce el 21 de marzo.

A finales de mayo o junio, el tiempo ha incidido en la chakra. Los granos tiernos que había hasta el mes de abril, aproximadamente, se han secado. El maíz, las habas, el fréjol, los chochos están secos y para muchos pueblos, especialmente del norte, constituyen motivo de grandes celebraciones como el hatun puncha, gran día, actualmente conocido como el Inti raymi, fiesta del sol. Gracias al tiempo seco, presencia del sol y del viento ha sido posible continuar con el ciclo de la vida, pues las semillas secas permitirán el ciclo de la siembra. Al

\footnotetext{
${ }^{5}$ Sembrío milenario de cultivo asociativo de semillas como el maíz, fréjol, chochos, quinua, papa, oca, mashua. 
tiempo seco se lo identifica como un tiempo fuerte, bravo, de carácter masculino. En los pueblos del norte del Ecuador constituye la celebración mayor del año.

\section{El valor estético de la lengua quichua}

En la lengua quichua tenemos partículas gramaticales o morfemas que contienen o recogen en la palabra el significado estético de la lengua; aquello se aprecia en las maneras respetuosas de relacionarse en el ayllu, especialmente con los yuyak. El respeto en la cultura quichua se evidencia en la relación con los mayores porque constituyen los guías de la vida; personas desprendidas de llevar una vida con apego a lo material. Son las personas que mejor entienden y practican el principio de relacionalidad andino y comprenden el valor cosmocéntrico de la vida. El respeto se extiende de la comunidad humana hacia la comunidad de las plantas, los cerros, las deidades, los difuntos, al cosmos; es una manera de hablar y accionar con cortesía, con amabilidad. Kinkushpa rimana, habla zigzagueada se encuentra en la tradición oral, como aquel concepto que guarda los códigos de relacionamiento de esta lengua.

\section{Kinkushpa rimana}

Kinkushpa rimana, es la forma zigzagueada de hablar; es hablar en zigzag. Kinku es la forma zigzageada que adoptan ciertas cosas, como los caminos, la forma de sembrar la chakra, por ejemplo. En los Andes esta forma de la siembra también se transmite al runa shimi, es decir idioma del ser humano, y donde encontramos la belleza del habla. Dirigirse, hablarles a los yuyak, los mayores, se enmarca dentro de esta dimensión estética, manifestada en el trato con respeto, autoridad, afectividad. El respeto en la cultura quichua se construye con base en la relación con los mayores porque constituyen los guías de la vida, como se mencionó.

En la estructura gramatical de este idioma encontramos partículas que ayudan a hablar y relacionarnos de forma educada, respetuosa, afectiva. La partícula -lla- se utiliza para denotar lo afectivo y respetuoso. Imanalla mamalla: ¿cómo está, mamita? La partícula -panos permite hablar con amabilidad, pedir las cosas de forma cortés, atenta. Shamupay mamalla: pase mamita, por favor. La partícula $-\boldsymbol{k} \boldsymbol{u}$ - es un morfema que expresa cariño, ternura. Shamupay mamakulla: pase mamita por favor, es un trato doblemente afectivo. El respeto connota un trato afectivo, tierno, considerado, que se manifiesta en el uso de estas partículas. Los jóvenes se encuentran ante el desafío de emplearas con eficiencia frente a los mayores o personas de autoridad.

\section{Minkachiway}

Es un saludo, que se emplea para anunciar la llegada del visitante. Minkachiway tiene mayor profundidad que la traducción al español puede ofrecer porque implica un "pedido de permiso", como muestra de respeto para poder llegar hacia la otra persona. El campo semántico de esta palabra parte de la voz minkana, que quiere decir encargar. Implica un 
pedido de 'hacerse cargo de', de forma amable. Los códigos culturales en palabras como esta demuestran que la cultura quichua otorga una importancia mayor a la relación con los demás, especialmente si son personas mayores o autoridades, ante las cuales se muestra un comportamiento que denota humildad, afecto, respeto.

Los quichuas pertenecen a una cultura donde se saluda mucho y se lo hace de diferentes maneras, dependiendo del contexto comunicacional en el que se exprese el saludo; en él se puede identificar la experiencia emocional que denota el acto comunicacional que una persona realiza para hacer notar su presencia. Al pasar frente a una casa se decía "yallichiway", déjeme pasar; o "ñankuta mañachiy", présteme el caminito. $\mathrm{O}$ al encontrarse con alguien en el camino: "ñachu shamukupanki", ¿ya está viniendo?; y al saludar a alguien que vemos ha pasado trabajando durante el día se decía kaynakupankichu, ñachu tukuripan: ¿está pasando el día?, ¿ya termina la jornada? Kawsakupankichu es un saludo que en la traducción al castellano equivaldría a decir ¿estás viviendo? Este saludo denota la profundidad del pensamiento andino, pues implica tener conciencia y dar valor al hecho de estar vivo; en respuesta al saludo, decimos de forma afectiva Ari, kawsakupanimi, ¡Sí, estoy viviendo! En este saludo se encuentra implícito un estado emocional personal interno que denota alegría de ver al otro con vida, y de la misma manera, alegría de estar con vida:

"Mientras más cariñoso y expresivo era mi saludo, mayor era el aprecio que recibía de la otra persona". "A las personas que saludan con respeto, los mayores en la comunidad suelen decir: Chay runaka shimi huntawan saludan" (A. Medina, comunicación personal, 30 de septiembre de 2017).

Lo anterior, equivale a decir que esa persona 'con boca llena saluda'; es decir que saluda con ternura, con respeto.

\section{Estados de ánimo y condiciones psíquicas del runa en la lengua}

Con la voz shunku se construye la "riqueza de sus derivaciones para la denominación de los diferentes y variados estados de ánimo y condiciones psíquicas”. (Golte, 1973a, 213). $\mathrm{Al}$ observar las diferentes construcciones semánticas realizadas por los abuelos con la palabra shunku, se constata el entendimiento de lo corpóreo dentro de una dimensión psicológica y espiritual.

\section{Shunkuyaypi/en la dimensión emocional}

- Alli shunku

- Mana alli shunku

- Upa shunku

- Wawa shunku

- Millay shunku

- Chushak shunk buen corazón, bondadoso, generoso

mal corazón, de no confiar

corazón mudo, inocencia

corazón de niño, inocencia

corazón bravo, valentía

vacío de corazón, de sentimientos, el que no es recíproco 
DOI: https://doi.org/ 10.20396/zet.v26il.8650878

- Shunku ukulla

- Ishkay shunku

- Shunku illak

- Api shunku

- Rumi shunku

- Runa shunku

- Piña shunku

- Shunkushka adentro del corazón, en silencio, en el interior

dos corazones, desleal, mentiroso

sin corazón, mezquino, el que no comparte

corazón blando, el de fácil llanto

corazón de piedra, fuerte, decidido

corazón humano andino

corazón bravo, de mal carácter

caprichoso

\section{Aycha ukupi/en la dimensión corpórea}

En la dimensión corpórea, shunku se denomina "las entrañas del cuerpo humano, en especial el corazón y el estómago. Esta región del cuerpo es en el pensamiento quechua el lugar en el cual se localizan las emociones, la razón y la memoria” (Golte, 1973b, 213).

- Yurak shunku, parak

- Yana shunku

- Shunku

- Maylla shunku

- Shunkuta tikrachi

- Shunkunanay

- Shunkullina, shunkuna

- Shunkuna pulmón

hígado, donde se encuentra la fuerza vital

corazón

corazón limpio, en ayunas, en sano juicio

devolver el corazón, vómito

dolor del corazón

desayunar

desayunar

\section{Willkaypi/en la dimensión espiritual}

- Shunku urmashka, mancharishka

- Shunkuchina corazón caído, espantado

hacer corazón, curar del espanto

Shunku es una palabra maravillosa porque a través de su campo semántico podemos entender que la salud o la enfermedad no solomente connotan una condición corpórea, física del cuerpo humano, sino que tienen también una relación con lo psíquico y lo espiritual. La enfermedad no puede ser tratada solamente en su dimensión material, se debe curar además el corazón, la mente y el espíritu, por medio de rituales como el shunkuchina, donde el cuerpo tiene que recuperar sus vibraciones y emociones, para restablecer el equilibrio del sami, la energía. Las vibraciones, los sonidos, son otros de los lenguajes para conectarnos a través del shunku con la pacha, los apukuna, cerros, los ayakuna, espíritus. La voz shunku supera los 
significados de la dimensión material, lo corpóreo para construir sentidos dentro de la dimensión emocional: los estados de ánimo, lo espiritual; manifiesta una comprensión del Ser-Runa dentro de una dimensión integral, holística.

\section{El conocimiento de tipo matemático en la lengua quichua}

Se cree que las matemáticas son independientes de las culturas y sociedades en que se desarrollan, que son de carácter universal; sin embargo, tienen una relación directa con la cultura y dentro de ella con las lenguas. El conocimiento de tipo matemático de la cultura quichua, actualmente, se evidencia en el desarrollo concreto de reglas de ordenamiento o clasificación, como el ishkaychi, conocimiento y comprensión sobre el ordenamiento de los números; yupana y sus aplicaciones como yapachina, mirachina: aumentar, anchuchina: quitar, rakina: dividir. La comprensión de las nociones de espacio también tiene su desarrollo en la cultura quichua, como hemos visto en la voz pacha. Esto nos conduce a entender el pensamiento matemático andino desde una lógica cosmocéntrica, "cada sociedad selecciona y desarrolla unas operaciones cognitivas más que otras en función de las necesidades que plantean su medio ambiente y su cultura" (Romero y Gottret, 2001).

Los sistemas de representación y escritura quichua muestran el desarrollo de léxicos especializados por y para la utilización de la matemática, como lo encontramos en los números que tienen sistemas sonoros propios. De la misma manera, el aprendizaje de la matemática en los niños y niñas quichuas no es independiente de la lengua natural, en este caso de la lengua quichua, de allí la importancia de la escuela como espacio para abordar este tipo de conocimientos desde sus propios léxicos matemáticos.

Si bien el lenguaje matemático quichua tiene un léxico concreto desarrollado, no es de uso específico de expertos, es un lenguaje comprensible, cotidiano, de carácter aplicativo para la solución de problemas contables, administrativos, de medición que se presentan en el día a día.

El alfabeto quichua se encuentra desarrollado con préstamos del castellano. A partir del Programa Nacional de Alfabetización se logró la oficialización del alfabeto quichua a fin de acceder a la escritura unificada, que hasta 2004 registraba varios intentos de estandarización del alfabeto. Particularmente en 1980 se definieron 21 grafías; luego, en 1998 se incorporó la "h", en lugar de la "j" y la posibilidad de utilizar "ts". Actualmente, la representación gráfica de los números se realiza con la escritura internacional. El conocimiento de tipo matemático en la cultura quichua al momento se hace evidente en el desarrollo concreto de reglas de ordenamiento o clasificación, como el ishkaychina ${ }^{6}$,

\footnotetext{
${ }^{6}$ Clasificar en dos elementos. 
conocimiento y comprensión sobre el ordenamiento de los números; el yupana ${ }^{7}$ y sus aplicaciones como yapachina ${ }^{8}$ : aumentar, anchuchina ${ }^{9}$ : quitar, rakina ${ }^{10}$ : dividir.

Al hacer una aproximación al conocimiento matemático andino a través del estudio de la lengua, podemos profundizar en cómo se desarrollan las comparaciones, organización, conformación de conjuntos, sistemas de numeración, desarrollo de operaciones. Afortunadamente, en la lengua quichua se han guardado los símbolos sonoros de tipo matemático y con ello su uso concreto en la realidad; la lengua, al cumplir el rol social de comunicar un sistema de signos, logró transmitir estos conocimientos, pero es fundamental continuar con el estudio para identificar los símbolos gráficos que antes se desarrollaron para la abstracción de este tipo de conocimientos.

\section{Sistema de numeración desde el quichua}

En la cultura quichua, al igual que las del mundo, se abordó la necesidad de contar, medir y determinar la forma de todo lo circundante, de allí que encontramos un conjunto de conocimientos numéricos y geométricos en uso hasta la actualidad. Igual que en el castellano "los niños que llegan a la escuela traen consigo un conocimiento matemático informal" (Hernández, López, Quintero y Velásquez, 2015). El sistema de numeración quichua es de base diez, es un sistema de representación decimal; se cuenta así: shuk (1), ishkay (2), kimsa (3), chusku (4), pichka (5), sukta (6), kanchis (7), pusak (8), iskun (9), chunka (10).

Ahora debemos explicarnos ¿cómo se genera el orden de conteo a partir de esta base decimal numérica? El conteo se produce sumando, y a partir de allí es posible la representación infinita de cantidades numéricas y realizar complejas operaciones; chunka shuk, diez más uno (11); chunka ishkay, diez más dos (12); chunka kimsa, diez más tres (13), chunka chusku; diez más cuatro (14); chunka pichka, diez más cinco (15); chunka sukta, diez más seis (16); chunka kanchis, diez más siete (17); chunka pusak, diez más ocho (18); chunka iskun; diez más nueve (19) e ishkay chunka, dos veces diez (20). Para continuar contando y construyendo el orden numérico de las decenas sumamos la decena chunka (10) más la unidad y determinamos la cantidad que queremos representar. Tres veces 10 , kimsa chunka (30); cuatro veces diez, chusku chunka (40); cinco veces diez, pichka chunka (50); seis veces diez, sukta chunka (60); siete veces diez, kanchis chunka (70); ocho veces diez, pusak chunka (80), nueve veces diez, iskun chunka (90). Para transformar las decenas solamente se añaden las unidades, y contamos: dos veces diez más uno, ishkay chunka shuk (21); tres veces diez más dos, kimsa chunka ishkay (32); cuatro veces diez más cuatro, chusku chunka chusku (44) ...nueve veces diez más nueve, iskun chunka iskun (99).

De esta forma llegamos a la centena, patsak (100); al millar, waranka (1000); al millón, hипи (1.000.000). Sin duda, en el conteo, yupay, de la cultura quichua se manifiesta

\footnotetext{
${ }^{7}$ En quichua significa número.

${ }^{8}$ En quichua significa aumentar.

${ }^{9}$ En quichua significa quitar.

${ }^{10}$ En quichua significa dividir. 
DOI: https://doi.org/ 10.20396/zet.v26il.8650878

un proceso de orden de los números sorprendentemente lógico, a través del cual apreciamos la elaboración de un pensamiento matemático que desarrolló habilidades de razonamiento para comprender conceptos dentro de esquemas de orden distintos de los de otras lenguas como el castellano, por ejemplo. Dentro de este contexto cabe preguntarnos ¿cómo se entendió el cero (0) en la cultura quichua?

Illak: cero (0)

En la lengua quichua tenemos la palabra illak para designar lo inexistente. Actualmente, dentro de la numeración quichua a la palabra illak se le atribuye un significado equivalente al cero (0) del castellano; sin embargo, su valor no se podría entender de forma unidimensional, pues la inexistencia como tal está determinada por la temporalidadespacialidad del universo. Illak expresa: "1. lo inexistente, 2. Carente, sin nada. 3. Ausente" $"$, es decir nos da a entender como aquello que no está ocupando un espacio, un lugar y un tiempo.

\section{Mashnaykuna: cantidades}

La asignación de cantidades, de magnitud matemática (numérica), están desarrolladas según las propiedades de los elementos (conjuntos medibles). Por ejemplo: tawka y achka significan "bastantes, bastante", respectivamente, su uso dependerá de la propiedad medible del conjunto, finito e infinito, como se explicita en la siguiente tabla 1:

Tabla 1: Mashnaykuna: cantidades

\begin{tabular}{cl}
\hline Cantidades & \multicolumn{1}{c}{ Propiedades del conjunto } \\
\hline Asha & poco (cosas infinitas) Asha kachita apamuy: Trae un poco de sal. \\
& Asha sarata apamuy: Trae un poco de maíz \\
Pishi & poco (cosas infinitas) Pishi kachita apamuy: Trae un poco de sal. \\
& Pishi sarata apamuy: Trae un poco maíz. \\
Achka & bastante (cosas infinitas) Achkata mikunki: Comes bastante. \\
& Achka kuyllurkunata rikuni: Miro bastantes estrellas. \\
Tawka & mucho (cosas finitas) Tawka warmikuna tiyan: Hay muchas \\
& mujeres. \\
\hline
\end{tabular}

Fuente: de la Autora.

\section{Asignaciones de cantidad por propiedades medibles}

\section{Chimpapurachi, comparaciones}

La partícula -pura permite establecer relaciones de similitud y equivalencia entre dos o más elementos. Paypura, entre semejantes:

Paypura sarakunata tantachiy: Recoge los maices semejantes.

Ñukanchikpura kawsanchik: Vivimos entre semejantes.

${ }^{11}$ Shimiyukkmu Diccionario kichwa-español, español-kichwa. 
DOI: https://doi.org/ 10.20396/zet.v26il.8650878

Se establecen relaciones de comparación numérica con los adjetivos de cantidad yalli, ashtawan, pishi, asha:

- Yalli: más, menos, demás, demasiado.

Kanka ñukata yalli purinki: Tú caminas más que yo.

Saratami purututa yalli tarpuna: Sembrar más maíz que fréjol.

Kunturmi ankakuna yalli hatun kan: El cóndor es más grande que el gavilán.

- Pishi, asha: poco, escaso.

Chilinaka chiwillata yalli pishi mishkimi kan: La naranja es menos dulce que la piña.

Kanka ñukata yalli pishillata purinki: Tú caminas menos que yo.

Kanka ñukata yalli ashallata purinki: Tú caminas menos que yo.

\section{Cantidades próximas a través de la partícula -lla}

La partícula -lla permite identificar cantidades próximas, no necesariamente exactas; significa: alrededor de, aproximadamente.

- Yapa (aumento)

- Yalli (más)

- Asha (poco)

- Pishi (poco) yapalla Yapalla kullkita charini: Tengo más o menos una cantidad de dinero.

yallilla Yallilla puchkata puchkani: Ovillo una cantidad más o menos de hilo.

ashaklla Ashakllata purishkani: He caminado apenas un poco.

pishilla Pishilla sarata munani: Quiero un poco de maíz.

\section{Conclusiones}

Las lenguas ancestrales, en este caso el quichua, anidan en sus significados aprehensiones del mundo que denotan una particular especialización del conocimiento; para explorar las lógicas de la agricultura, medicina, arquitectura, matemática, filosofía, valores, estados emocionales, comprensión de sociedad, la lengua y el estudio del campo semántico constituyen las herramientas para profundizar en los significados. Considerar a las lenguas ancestrales como nidos de la diversidad del pensamiento, sitúa a los seres humanos portadores de ellas como sujetos activos en la construcción y resolución de los problemas de la sociedad moderna; los reconoce, visualiza y pone en valor como sujetos pensantes, con derecho a decidir sus destinos. 
DOI: https://doi.org/ 10.20396/zet.v26il.8650878

Es trascendente para la pervivencia de todas las formas de existencia, dedicar tiempoespacio y esfuerzos a documentar la forma de pensar y reflexionar frente a la vida que mantienen en vigencia los pueblos ancestrales de los Andes, a fin de poner en práctica esos conocimietos y sabiduría en todos los niveles de la sociedad. El sistema educativo de las sociedades modernas debe asumir este reto y contribuir de forma activa a la interculturalización de los Estados con la presencia de las diversidades culturales y lingüísticas; con ello surge la necesidad de renovar el currículo, de tal manera que, por fin, esté orientado atender las necesidades educativas de culturas marginalizadas.

Es necesario globalizar los conocimientos y sabidurías de las culturas ancestrales, pues en ellas se encuentra una comprensión de la vida respetuosa, amable con el cosmos. Es derecho de todos los niños y niñas del mundo aprender en las familias y las escuelas formas antiguas de relacionarse con lo circundante, donde culturas como la quichua entienden que todo está vivo, Tukuymi kawsa, esto como forma de poner en resguardo la sobrevivencia de la humanidad frente a la amenaza de prácticas de vida consumistas e individualistas en las que se encierran las sociedades actuales.

\section{Referencias}

Cachiguango, L. (2007). PAKARINA: El ritual andino del nacimiento de la crianza de niños y niñas en Cotacachi. Cotacachi: Cruz Roja Ecuatoriana.

Hernández, O., López, J., Quintero, A., y Velazquez, A. (2015). El sentido numérico: más allá de los números. Puerto Rico.

Golte, J. (1973). El concepto de SONQO en el RUNA SIMI del siglo XVI. (213-217). Indiana. V.1, (pp.213-218).

Romero, R., \& Gottret G. (comp). (2001). "Matemática andina: Abordaje psicogenético". En A. E. Lizarzaburu \& G. Zapata Soto, Pluriculturalidad y aprendizaje de la matemática en la América Latina. Experiencias y desafíos (Morata S.L, pp. 125-153). Madrid.

Schaff, A. (1967). Lenguaje y conocimiento. Mexico, D.F., Mexico. Editorial Grijalbo. S. A.

Moreno Cárdenas, F. H. (Org.). (2008). Shimiyukkmu, Diccionario kichwa-español, españolkichwa. Quito: Casa de la Cultura Ecuatoriana Benjamín Carrión de Sucumbíos.

Recebido em: 31/10/2017

Aprovado para publicação em: 03/04/2018 